\title{
Síndrome de Burnout en Docentes de las Escuelas Normales del Estado de Chihuahua, México
}

\author{
Gabriela Vázquez Delgado \\ Benemérita y Centenaria Escuela Normal del Estado de Chihuahua \\ gabriela.prf68@hotmail.com \\ Rosalva Flores Zubía \\ Benemérita y Centenaria Escuela Normal del Estado de Chihuahua \\ rosalvafloreszubia@hotmail.com \\ Anabell Sosa Fierro \\ Benemérita y Centenaria Escuela Normal del Estado de Chihuahua \\ anabellsofi@hotmail.com
}

\section{Resumen}

El objetivo principal de esta investigación es identificar la presencia del síndrome de Burnout o desgaste profesional y ocupacional (SAD) en profesores de las escuelas normales del estado de Chihuahua. Burnout es una condición que se manifiesta con presencia de estrés (etapa avanzada) en el cuerpo, debido a factores emocionales e interpersonales que se producen en el trabajo. Se utilizó estadística descriptiva (análisis de la varianza). La muestra del estudio es intencional, participando profesores de dos escuelas normales en la primera, una muestra de 55, que representan el $60 \%$ y en la otra normal 32 profesores, un 62\% de la población. Los instrumentos utilizados fueron una encuesta electrónica (Authorware 4.0), que incluye: MBI (Maslach Burnout Inventory), la historia clínica y un cuestionario sociodemográfico. El MBI consta de 22 ítems que comprenden tres subescalas: agotamiento emocional, despersonalización y realización personal. La historia clínica se integra por antecedentes personales, familiares y su cuestionario sociodemográfico que abarca los aspectos biológico, social, laboral y motivacional. Los resultados parciales permiten observar que la gran mayoría de los profesores encuestados afirman sentir un agotamiento emocional en nivel bajo. Es importante señalar que el análisis llevado a cabo hacer notar que el momento de la aplicación es la clave para arrojar los resultados. Podemos concluir entonces, que los profesores estudiados, en general, presentan síntomas actuales de burnout y los resultados se puede ver en los límites que indican su presencia, por lo que sugiere que se realicen acciones de impacto para el manejo del estrés.

Palabras clave

Burnout, Educación Superior, Docentes 


\section{Introducción}

El síndrome de Burnout, también conocido como síndrome de desgaste profesional, ocupacional (SDO) o incluso síndrome de quemarse por el trabajo. Maslach, C. y E. Jackson.(1986). Es considerado un padecimiento que se manifiesta en la presencia de estrés (fase avanzada) en el organismo, ante factores estresantes emocionales e interpersonales que se presentan en el trabajo. Puede observarse en el ámbito profesional y por lo tanto en la calidad de vida.

En la Normal de Chihuahua, un aspecto que llama particularmente la atención es que desde hace algunos años se ha dado una fuerte movilidad laboral por jubilaciones y a su planta docente han ingresado maestros que solo han permanecido uno o dos semestres. Estos eventos no se han documentado, es decir no se han estudiado las causas de su corta permanencia.

De manera informal se especula que es la multiplicidad de tareas que se realizan. Se reconoce que las condiciones de trabajo de los maestros de educación básica y las de los docentes de nivel superior, específicamente de las Normales, son muy distintas.

Aunado a lo anterior se observa una incidencia en el absentismo de algunos docentes ocasionado por incapacidades debido a accidentes o enfermedades que en muchas ocasiones tienen origen en el estrés.

En algunos artículos de Hiebert y Farber (1984) pueden encontrarse evidencias para considerar a la docencia como una profesión estresante.

Dworkinn, (1987); Farber, (1991) Señalan que las principales consecuencias que afectan a los docentes en su ámbito de trabajo son: la incapacidad para desconectarse del trabajo, los problemas de sueño, el cansancio aumenta la susceptibilidad a la enfermedad, problemas gastrointestinales, de espalda, cuello, dolores de cabeza, entre otros. Que están relacionados en ocasiones con el abuso de fármacos, tranquilizantes, estimulantes, abuso de café, alcohol y drogas.

Algunos síntomas más específicos se ven manifestados en cuatro áreas: psicosomáticas (fatiga crónica, trastornos del sueño, desordenes gástricos y tensión muscular) se caracteriza por una ausencia o falta de energía, entusiasmo y sentimiento de escasez de recurso a estos sentimientos pueden sumarse los de frustración y tensión en los trabajadores que se dan cuenta que ya no tienen condiciones de gastar más energía, otros de ellos es considerado de conducta donde una persona se ausenta de su puesto en horarios de trabajo, también se puede considerar algunas adicciones del tabaco, alcohol, entre otras. En el área emocional se puede observar irritabilidad, poca concentración, distanciamiento afectivo y por último el área laboral del trabajador a autoevaluarse de forma negativa. Las personas se sienten insatisfechas con su desarrollo profesional, experimentan una declinación en el sentimiento de competencia y de éxito en su trabajo y en su capacidad de interactuar con las personas.

Al pretender investigar si el personal docente de las Normales del Estado de Chihuahua presenta el Síndrome de Burnout, se aprecia que las condiciones laborales son muy distintas, pues las instituciones tienen una organización diferente, por lo que se descarta el estudio en la Escuela Normal Rural "Ricardo Flores Magón" de Saucillo y la Escuela Normal Particular Yermo y Parres de Creel, ambas con la modalidad de internado y sólo se analiza al personal de la Normal "Ricardo Flores Magón" de la ciudad de Parral, Chihuahua y la Benemérita y Centenaria Escuela Normal del Estado de Chihuahua.

\section{Propósitos}

- Explorar las manifestaciones de desgaste emocional $y$ físico $\mathrm{o}$, 
síndrome de Burnout, en los docentes normalistas.

- Conocer las variables sociodemográficas que afectan el desempeño del personal docente de la institución.

- Contribuir al establecimiento de un clima de crecimiento y aprendizaje en la planta docente de la institución.

- Proponer estrategias viables para apoyar el crecimiento emocional y desarrollo humano del personal docente, que impacte en su práctica

\section{Metodología}

Para medir el Burnout en docentes se aplica una encuesta en versión electrónica (Authorware 4.0), misma que incluye: el MBI (Maslach Burnout Inventory), historia clínica y un cuestionario sociodemográfico.

El análisis se realiza a partir de la estadística descriptiva.

\section{Instrumentos}

El MBI es un instrumento de evaluación, en donde se valoran tres dimensiones a través de tres subescalas. Consta de 22 ítems las cuales incluyen: afirmaciones sobre pensamientos, sentimientos, emociones y conductas del profesional relacionados con su trabajo (Maslach C y Jackson SE, 1981).

Las subescalas están especificadas así:

Agotamiento o cansancio emocional, la descripción de esta subescala se define como la respuesta que manifiesta el individuo ante condiciones que se le presentan en su vida cotidiana y resultan desfavorables, de tal forma que sobrepasan sus recursos para dar respuesta a esa situación de manera adaptable.

Despersonalización: Se considera como la transformación sobre la percepción que se tiene de uno mismo; de tal forma que el individuo se siente "separado" de los procesos mentales o de su cuerpo.
Realización personal: Se define como la forma en que uno se percibe, con la autenticidad, con ser lo que se decide ser y no con lo que se aprende o se construye de forma social o cultural.

En nuestro país Moreno y colaboradores en 1991 hicieron una versión en castellano del MBI y los estudios sobre el síndrome se han realizado en Universidades Mexicanas; ejemplo de ello son: Factores psicosociales y síndrome de burnout en docentes. Universidad del valle de Atemajac de Pando Manuel y cols.(2006), Desgaste laboral en profesores de secundaria de la ciudad de México. Elizalde (2010) y Factores psicosociales laborales y síndrome de Burnout, diferencias entre hombres y mujeres docentes de secundaria. Aldrete y cols. (2008).

Dado que el MBI puede aplicarse fácilmente, ha sido utilizado en personal que está en contacto con servicios humanos como maestros, médicos, psicólogos, trabajadores sociales, entre otros.

Sobre la historia clínica se puede decir que los datos clínicos incorporan sus antecedentes personales, familiares y sus hábitos. En este caso la historia clínica no se limita a ser una exposición de hechos simplemente, sino que contiene una sección de información en donde se incluyen los siguientes cuestionamientos: ¿Padece dolores de cabeza?, ¿Tiene pérdida de apetito?, ¿Tiene alteraciones en el sueño?, ¿Presenta ansiedad?, entre otros.

El cuestionario sociodemográfico engloba los aspectos:

- Biológicos y sociales: donde se incluye el género, la edad, estado civil, número de hijos y más.

- Laborales: Años de experiencia, categorías laborales, número de horas frente a grupo entre otros.

- Motivacionales: factores de satisfacción e insatisfacción en la docencia 


\section{Sujetos}

Las características que se consideraron para llevar a cabo la investigación fueron:

- Docentes frente a grupo

- Directivos

De la aplicación que se lleva a cabo en la IByCENECH se toma en consideración para el estudio a 81 docentes, hombres y mujeres de los cuales solo 52 de ellos responden la encuesta, tenemos entonces que el $64 \%$ de la población de docentes participa en la investigación, de la cual $73 \%$ son mujeres y hombres $26 \%$. En otro momento se asiste a la
Normal Experimental Miguel Hidalgo ubicada en la Ciudad de Parral, Chihuahua; en donde el total de la población consta de 34 sujetos, de los cuales 21 corresponden a la muestra, quedando cubierto el $62 \%$ de la población, por razones de estudio en este apartado se ilustra el análisis detallado de los datos estadísticos y se presenta como ejemplo, los resultados con los docentes de la IByCENECH.

Del análisis estadístico se obtiene la siguiente información:

\section{Resultados preliminares}

Subescala de agotamiento emocional

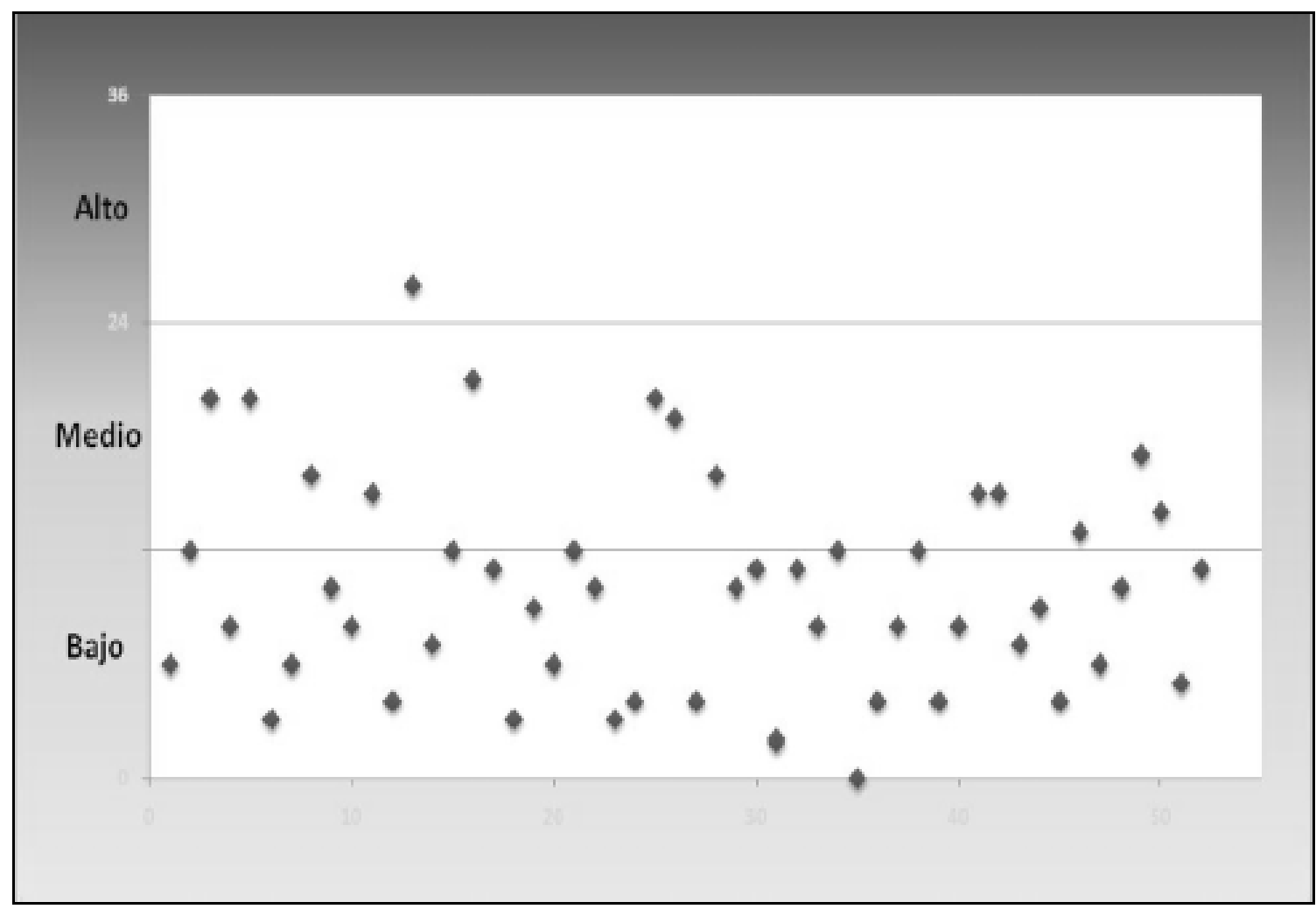

Gráfica 1. Dispersión del total de docentes de la IByCENECH, de acuerdo a los niveles de agotamiento emocional.

Como se aprecia en la gráfica 1, la gran mayoría de los docentes dicen sentir un nivel de agotamiento bajo, y sólo el 26 \% expresó estar en un nivel medio de agotamiento emocional, más sólo una persona resulta en el nivel alto. 


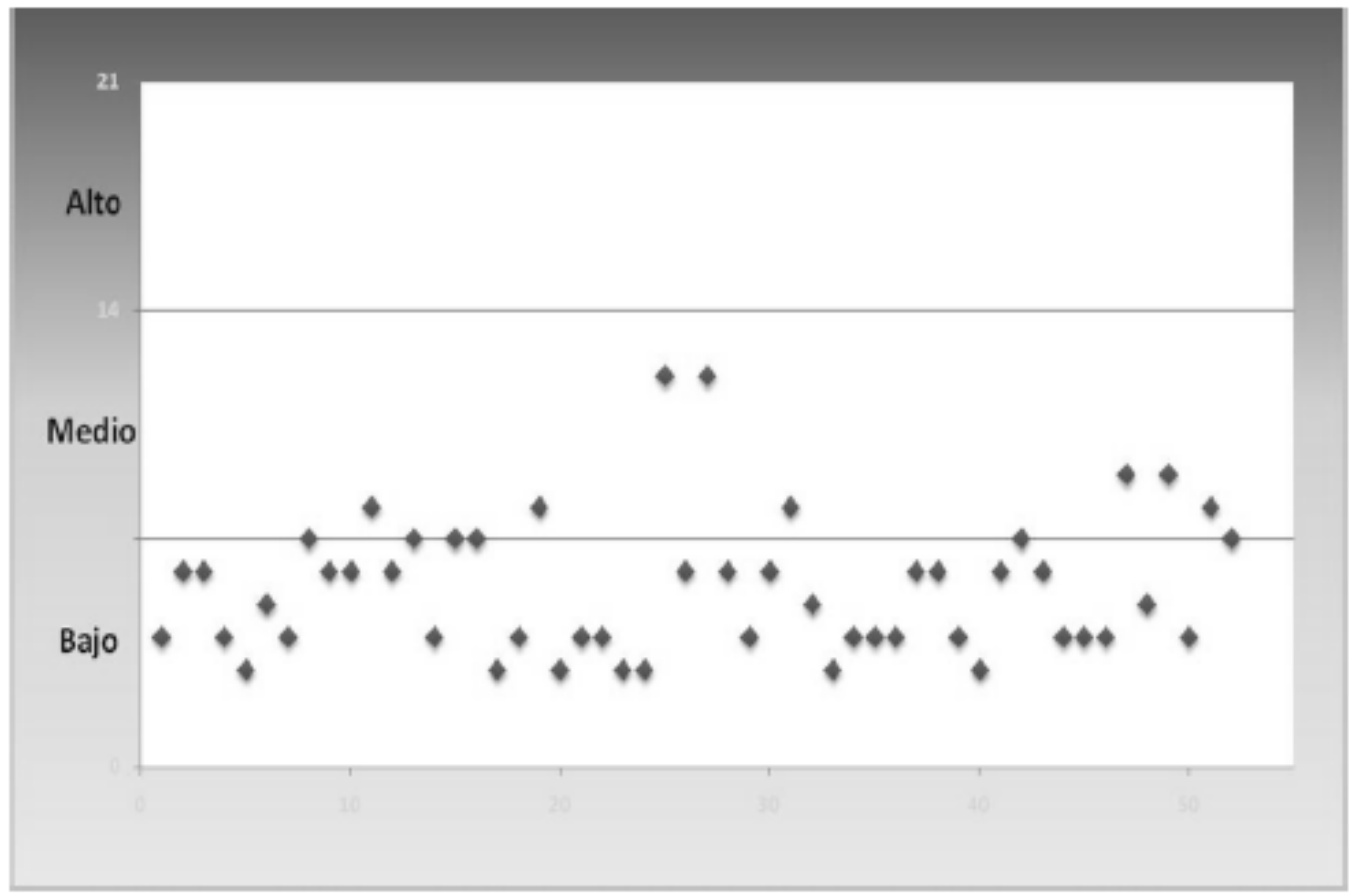

Gráfica 2. Dispersión del total de docentes de la IByCENECH con respecto al nivel de despersonalización.

La gráfica 2 nos muestra que sólo el 24\% de los docentes de la institución dicen presentar algún signo característico de la despersonalización, situación que se explica a partir de que la profesión docente se enclava en el área de humanidades.

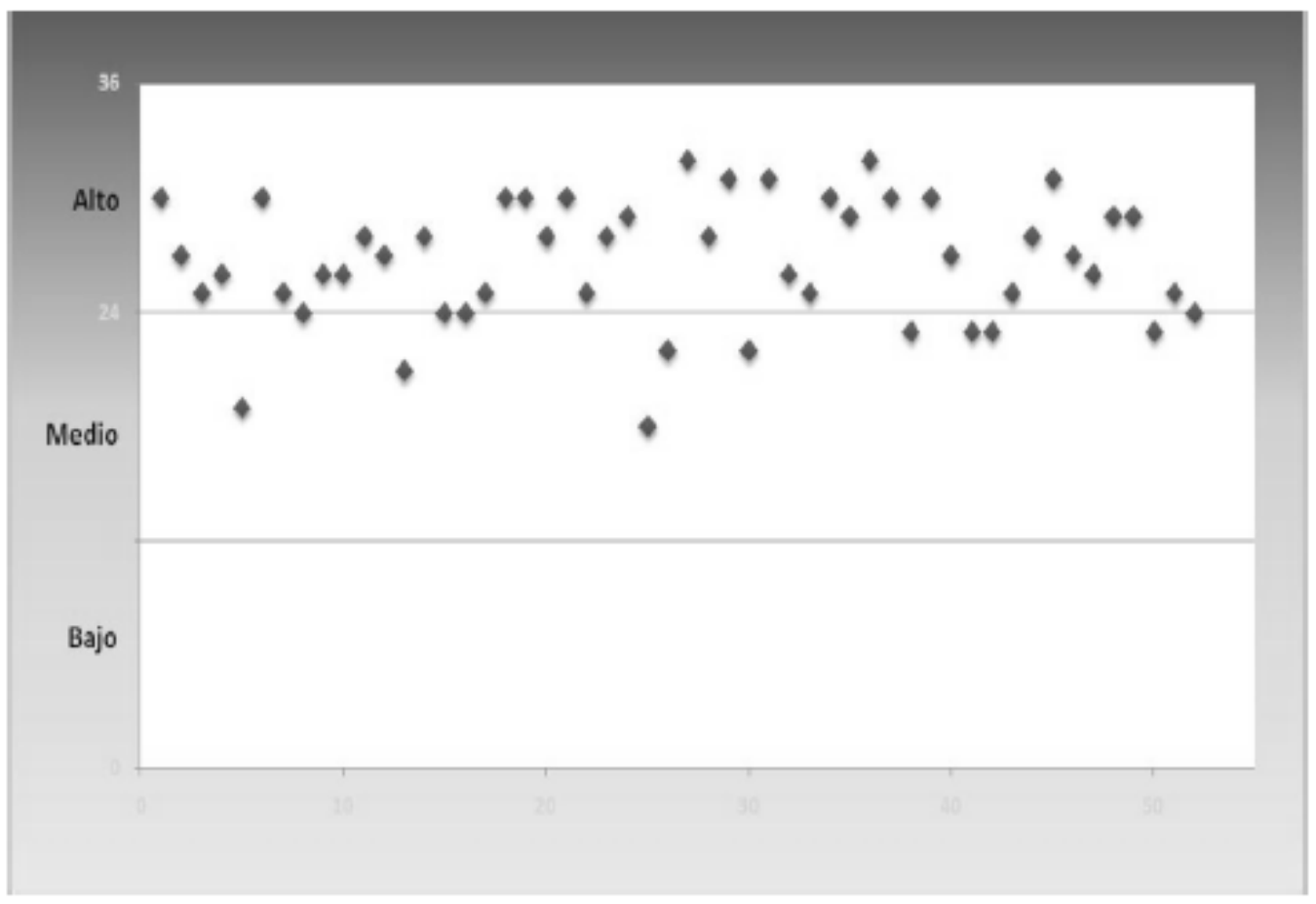

Gráfica 3. Dispersión del total de maestros de la IByCENECH, ubicados en el nivel de realización personal. 
El resultado de la realización personal es muy satisfactorio ya que los maestros se proyectan en un nivel alto como puede observarse en el gráfico 3.

Una de las sublíneas sobre las cuales se trabaja en este estudio es con relación al estudio de género. En las investigaciones en ciencias sociales ha tenido una gran relevancia, y hoy nos ocupa en un sector específico, como es en el educativo; por tradición, un gran número de aspirantes a ingresar a la educación normal son mujeres "feminización docente", menciona González (2009) que en el 2009 se tenía un registro en donde el $76.2 \%$ de los docentes eran mujeres, un porcentaje interesante porque permite reflexionar el por y para qué de la elección de esta carrera de vida. Se ha comentado que es un trabajo cómodo, de medio turno, que es compatible con el ser madre de familia, que se tienen prestaciones, entre otras, $y$ es pertinente cuestionarse entonces, por qué las docentes mujeres de la Escuela Normal Experimental "Miguel Hidalgo" en Parral y la Escuela Normal del Estado en Chihuahua "Profr. Luis Urías Belderráin" (IByCENECH), manifiestan sentirse abrumadas con la carga laboral.

Al analizar los datos por género encontramos que la diferencia entre docentes mujeres y hombres no es significativa, sin embargo se aprecia que el género femenino presenta mayor puntaje en la escala de agotamiento emocional. Esto coincide con la literatura revisada (Greenglass y Burke, 1988. Hakanen, 1999).

Algunos estudios señalan que las mujeres trabajadoras tienen más probabilidades de padecer el síndrome de Burnout. En el Estudio de Marín y Paredes (2002) se encontró que el sexo y la edad están relacionados con el desgaste laboral.

Con respecto a la subescala de agotamiento emocional puntuaciones de $27 \mathrm{o}$ superiores indican un alto nivel de Burnout, entre 19 y 26 puntuaciones intermedias, siendo las puntuaciones por debajo de 19 indicativas de burnout bajo o muy bajo.

Al separar para el estudio a los docentes por género encontramos que los hombres presentan una media de 8 puntos, lo que los ubica en el nivel bajo de agotamiento, al igual que las mujeres docentes, quienes observan una media de 11 puntos esto se ilustra en el gráfico 5.

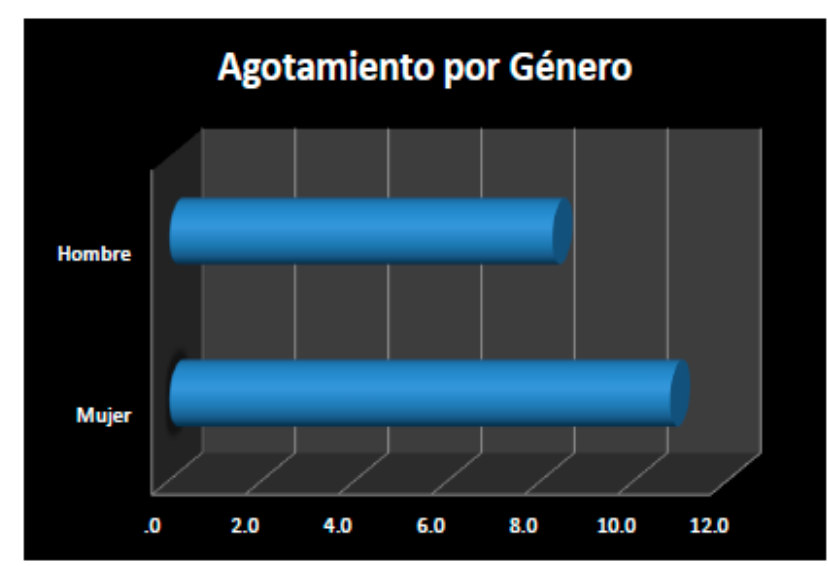

Gráfico 6. Puntajes promedio del agotamiento por género de docentes de la IByCENECH.

Aún cuando en el análisis estadístico no es significativo, se aprecia mayor agotamiento emocional en las docentes mujeres.

En la subescala de despersonalización puntuaciones superiores a 10 sería alto, de 6 a 9 medio y menor de 6, bajo grado de despersonalización.

El análisis de los datos señala que los docentes varones obtienen un puntaje promedio de 5.5, mientras que el género femenino, puntaje similar, 5.1. Ambos refieren un grado bajo de despersonalización. 


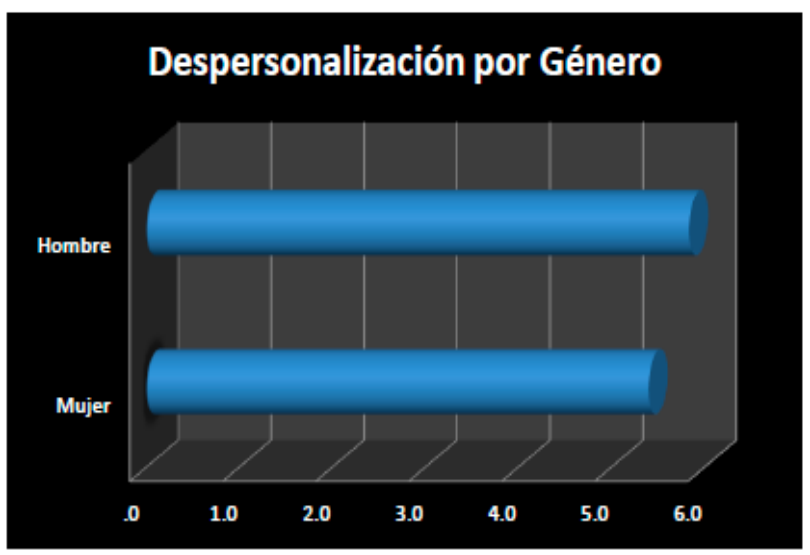

Gráfico 7 Puntaje promedio de despersonalización en docentes.

En lo que respecta a la subescala de realización personal, el puntaje funciona de manera inversa a las anteriores de 0 a 30 indicaría baja realización personal, de 34 a 19 intermedia y superior a 40 sensación de logro.

Los datos analizados permiten advertir que los docentes hombres presentan un puntaje promedio de 14 puntos, mientras que las mujeres alcanzan un promedio de 17 puntos. Ambos se ubican en el nivel bajo de realización personal. De acuerdo con Rubio (2004) un bajo nivel de realización se manifiesta con sentimientos de incompetencia profesional. Ver gráfico 8.

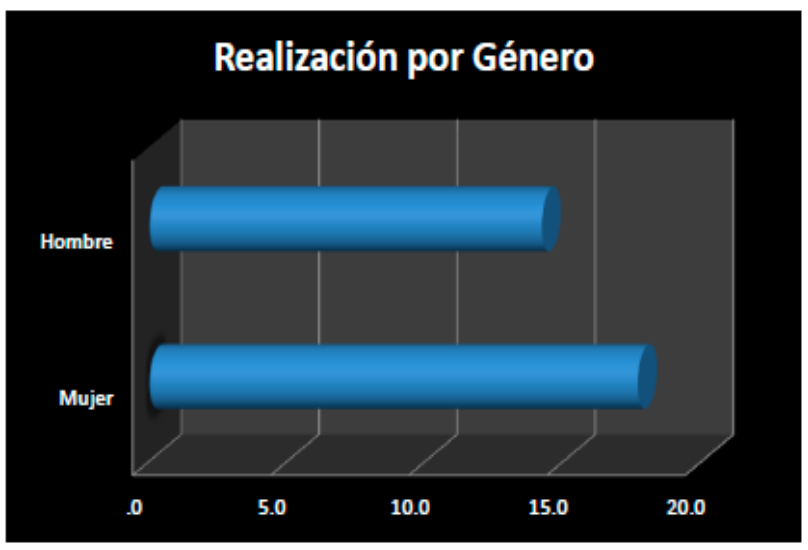

Gráfico 8 Puntajes promedio de realización personal de docentes.

\section{Conclusiones}

Podemos concluir entonces, que de manera general los docentes estudiados no presentan el síndrome de Burnout, sin embargo cerca del $20 \%$ de los docentes presentan de manera individual el síndrome.

Los resultados indican que con respecto al análisis de los datos, en general, en la muestra global el nivel es medio bajo en sus tres subescalas o dimensiones, no obstante en el caso del agotamiento emocional en las mujeres, se aprecia un mayor puntaje el cual es consistente con la bibliografía revisada y referida en el estudio.

El que las mujeres obtengan unos resultados que impliquen menor bienestar psicológico, puede encontrar explicación en el hecho de que la mujer realiza una doble tarea de acuerdo con la influencia cultural. El cumplir con el rol de ama de casa y como profesionista.

El estudio pone de manifiesto la presencia del síndrome de burnout debido a que la profesión demanda la atención y ofrecimiento de servicios humanos directos.

Hasta el momento, los resultados preliminares permiten advertir la presencia de la variable temporalidad debido a que el instrumento se aplica en el periodo intersemestral, que es el lapso en el que los docentes se encuentran en capacitación y planeación de cursos. Este factor puede incidir en los resultados, por lo que se considera importante una nueva aplicación del instrumento durante la jornada de trabajo frente a grupo, que es cuando el docente se enfrenta a una multiplicidad de tareas. Asimismo, continúa el estudio con el diseño e implementación de estrategias.

\section{Referencias}

Aldrete M., Preciado M, Franco S., Pérez, Aranda C. Factores psicosociales laborales y síndrome de Burnout, 
diferencias entre hombres y mujeres docentes de Secundaria, zona metropolitana de Guadalajara México. 2008.

Álvarez, E. y Fernández, L. El Síndrome de «Burnout» o el desgaste profesional. I: Revisión de estudios. Revista de la Asociación Española de Neuropsiquiatría. 991, vol. 11, No39. España.

Dworkin, A. G. (1987), Farber, B. A. (1991) Citado en International Journal of Clinical and Health Psychology. Estrés y burnout en profesores. Moriana, J. A. Universidad de Córdova, España. 2004

Elizalde, Alejadra. (2010) Desgaste laboral en profesores de secundaria de la ciudad de México. González, Rosa María. (2009) Revista mexicana de investigación educativa. Estudios de Género en educación: una rápida mirada. versión impresa ISSN 14056666 RMIE v.14 n.42 México jul./sep. 2009. Sección temática: Género en educación.

Greenglass, E.R. y Burke, R.J. (1988). Work and family precursors of burnout in teachers. Sex differences. Sex Roles, 18, 3-4, 215-229.

Hakanen, J. (1999) Gender-role related differences in burnout. Työterveiset 2 . (Small workplace program of the Finnish Institute of Occupational Health ). Retreived September 1, 2000, from the World Wide Web: http://www.occuphealth.fi/ttl/tiedotu s/tyoterv/299engl/burnout7.htm

Hiebert y Farber citado en Traves y Cooper (1997). El estrés de los profesores. La presión de la actividad docente. Ed. Paidós. España

Marin, H., \& Paredes, M. (2002).Estudio del Síndrome de Burnout o desgaste profesional en los profesores de la Universidad de los Andes. Educere, Investigación. Año 6 No. 17. Recuperado demayo de 2008 en http://redalyc.uaemex.mx/redalyc/sr c/inicio/ArtPdfRed.jsp?iCve $=3560170$ 4

Maslach, C. y E. Jackson.(1986).The MBI Inventory ( $2^{\mathrm{a}}$ ed.) Palo Alto, CA

Moreno B, Garrosa E y González J.(2001). Evaluación específica de los procesos de Burnout: Burnout de profesorado y de enfermería. Revista Interamericana de Psicología Ocupacional. Madrid.

Pando Moreno, Manuel y col (2006) Factores Psicosociales y Síndrome de Burnout en docentes del Valle de Atemajac, Guadalajara. En Redalyc. Salud en Tabasco, septiembre- diciembre, Año/Vol $12 \mathrm{~N}^{\circ}$ 003. México.

Rubio, Jiménez Jesús Carlos. 2004. Fuentes de estrés, síndrome de burnout o actitudes disfuncionales en orientadores de IES. http://www.aidex.es/estres/burnouto rientadorJC.pdf. 07 de febrero de 2012. 